TRANSACTIONS OF THE

AMERICAN MATHEMATICAL SOCIETY

Volume 358, Number 9, September 2006, Pages 4021-4044

S 0002-9947(05)03791-8

Article electronically published on September 22, 2005

\title{
ON THE CAUCHY PROBLEM OF DEGENERATE HYPERBOLIC EQUATIONS
}

\author{
QING HAN, JIA-XING HONG, AND CHANG-SHOU LIN
}

\begin{abstract}
In this paper, we study a class of degenerate hyperbolic equations and prove the existence of smooth solutions for Cauchy problems. The existence result is based on a priori estimates of Sobolev norms of solutions. Such estimates illustrate a loss of derivatives because of the degeneracy.
\end{abstract}

\section{INTRODUCTION}

It is well known that the Cauchy problem is well posed for strictly hyperbolic equations. The situation is complicated for degenerate hyperbolic equations. In [2], Colombini and Spagnolo constructed a degenerate hyperbolic equation such that the Cauchy problem is not well posed. The equation has a simple form

$$
\partial_{t t} w-a(t) \partial_{x x} w=0 \quad \text { in } \mathbb{R} \times(0, T),
$$

where $a(t)$ is a nonnegative function in $(0, T)$. In fact, solutions do not exist for some smooth Cauchy data even in the distribution sense. Such an example clearly shows that certain conditions are needed for the well-posedness of the Cauchy problem for degenerate hyperbolic equations. So far, the conditions are imposed on the finite degree of the degeneracy. It is proved that the Cauchy problem is well posed for the effectively hyperbolic equations. For details and references, see [10]. Roughly speaking, the effective hyperbolicity means the degeneracy occurs with degree two. In [1], Colombini, Ishida and Orrú studied degenerate hyperbolic equations with coefficients depending only on time. They showed that the Cauchy problem is well posed for the finite degeneracy. When coefficients also depend on the space variables, it is not known whether the finite degeneracy is sufficient for the well-posedness. In 3], D'Ancona and Trebeschi studied degenerate hyperbolic equations with analytic leading coefficients. In [8], Ishida and Odai studied degenerate hyperbolic equations with explicit expressions for the degeneracy and proved the well-posedness. For the discussion on the infinite degeneracy, see [9]. Both of [8] and [9] are devoted to the study in the Gevrey class.

In the present paper, we shall study the Cauchy problem for the following hyperbolic equation in $\mathbb{R}^{n} \times(0, T)$ :

$$
\partial_{t t} w-K(x, t) \sum_{i, j=1}^{n} a_{i j}(x, t) \partial_{i j} w-\sum_{i=1}^{n} b_{i}(x, t) \partial_{i} w-c(x, t) w=f,
$$

Received by the editors April 16, 2003 and, in revised form, June 21, 2004.

2000 Mathematics Subject Classification. Primary 35L15, 35L80.

Key words and phrases. Degenerate hyperbolic equations, Cauchy problems.

The first author was supported in part by an NSF grant and a Sloan research fellowship.

(c) 2005 American Mathematical Society Reverts to public domain 28 years from publication 
where $\left(a_{i j}\right)$ is positive definite and $K \geq 0$ in $\mathbb{R}^{n} \times(0, T)$. This is a degenerate hyperbolic equation since $K$ is allowed to vanish. We shall assume

$$
\lambda I \leq\left(a_{i j}\right) \leq \Lambda I \quad \text { in } \mathbb{R}^{n} \times[0, T],
$$

and for $i=1, \cdots, n$

$$
\left|b_{i}\right| \leq C \sqrt{K}, \quad \text { in } \mathbb{R}^{n} \times[0, T],
$$

for some positive constants $\lambda, \Lambda$ and $C$. The condition (1.3) is referred to as the Levi condition.

We shall impose the following condition on $K$ : The set $\mathbb{R}^{n} \times(0, T)$ can be decomposed into finitely many domains such that the boundaries of those domains are uniformly space-like surfaces (in the sense we will describe) and that $\partial_{t} K$ has a fixed sign in each domain. To be specific, we suppose $\mathbb{R}^{n} \times(0, T)$ has the finite decomposition

$$
\mathbb{R}^{n} \times(0, T)=\bigcup_{l} \Omega_{l},
$$

such that $\partial_{t} K$ has a fixed sign in $\Omega_{l}$ for each $l$, i.e.,

$$
\partial_{t} K \geq 0 \text { or } \leq 0 \text { in } \Omega_{l} \text {. }
$$

For each $l$, we assume $\Omega_{l}$ is defined as a region bounded by two graphs over a domain in $\mathbb{R}^{n}$, i.e.,

$$
\Omega_{l}=\left\{(x, t) ; x \in D_{l}, t_{l}^{-}(x)<t<t_{l}^{+}(x)\right\},
$$

for some domain $D_{l} \subset \mathbb{R}^{n}$ with a piecewise smooth boundary and for some piecewise smooth functions $t=t_{l}^{ \pm}(x)$ with

$$
\begin{aligned}
& 0 \leq t_{l}^{-}(x)<t_{l}^{+}(x) \leq T \text { for } x \in D_{l}, \\
& t_{l}^{-}(x)=t_{l}^{+}(x) \text { for } x \in \partial D_{l} .
\end{aligned}
$$

We let $\Sigma_{l}^{ \pm}$be the two surfaces over $D_{l}$ defined by $t=t_{l}^{ \pm}(x)$. We also assume that for some positive constants $\eta_{0}, \eta_{1}$ and $\eta_{2}$ there hold for any $l$

$$
\left|\partial_{x} t_{l}^{ \pm}\right| \leq \eta_{0} \quad \text { on } \Sigma_{l}^{ \pm}
$$

and

$$
\begin{aligned}
\eta_{1} & \leq \frac{1}{\sqrt{1+\left|\partial_{x} t_{l}^{ \pm}\right|^{2}}}\left(1-\left(K \sum_{i, j=1}^{n} a_{i j} \partial_{i} t_{l}^{ \pm} \partial_{j} t_{l}^{ \pm}\right)^{\frac{1}{2}}\right) \\
& \leq \frac{1}{\sqrt{1+\left|\partial_{x} t_{l}^{ \pm}\right|^{2}}}\left(1+\left(K \sum_{i, j=1}^{n} a_{i j} \partial_{i} t_{l}^{ \pm} \partial_{j} t_{l}^{ \pm}\right)^{\frac{1}{2}}\right) \leq \eta_{2} \text { on } \Sigma_{l}^{ \pm} .
\end{aligned}
$$

Note that, with (1.8), (1.9) always holds if $K$ is small. It is easy to see by (1.9) that $\Sigma_{l}^{ \pm}$is space-like uniformly for (1.1). The finiteness in the decomposition (1.4) can be relaxed. See section 5 for the discussion.

We need to emphasize that we do not require $K$ to vanish up to finite orders. In other words, the equation (1.1) may be infinitely degenerated.

Now we illustrate the decomposition (1.4) with (1.6)-(1.7) by some figures for $n=1$. In Figure 1, the decomposition clearly satisfies (1.6)-(1.7). In Figure 2, and - indicate the sign for $\partial_{t} K$. Clearly, the region $\left\{\partial_{t} K \leq 0\right\}$ does not satisfy (1.6)-(1.7). However, by adding an extra ray parallel to the horizontal axis, the 
new decomposition satisfies (1.6)-(1.7), as shown in Figure 3. As we can easily see, the constant function for $t$ always satisfies (1.8) and (1.9). Hence the newly added boundary in Figure 3 satisfies all the hypotheses imposed on the decomposition. (We shall discuss more on the horizontal boundaries later.)

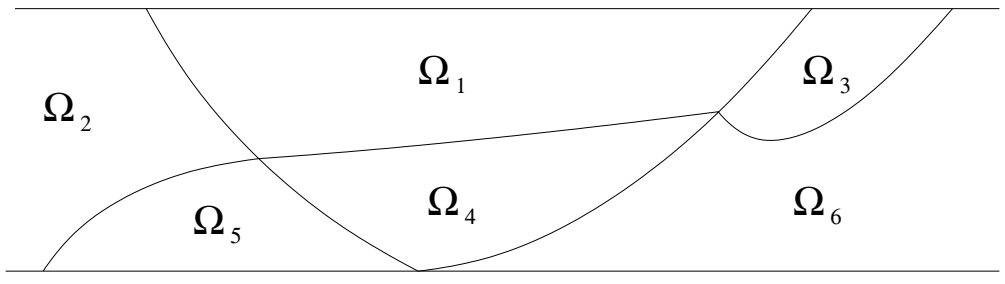

Figure 1. A decomposition that satisfies (1.6)-(1.7).

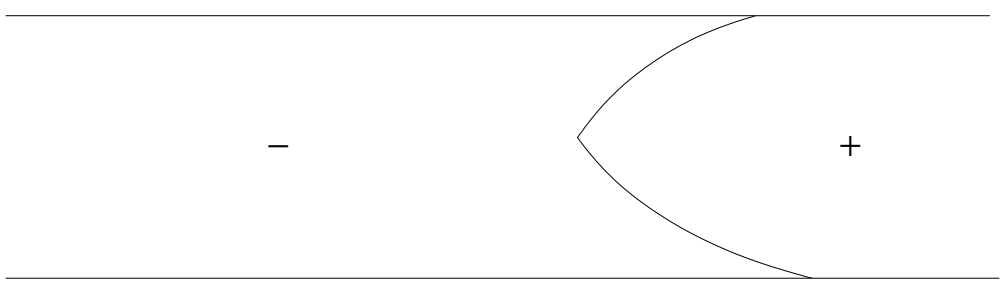

Figure 2. A decomposition that does not satisfy (1.6)-(1.7).

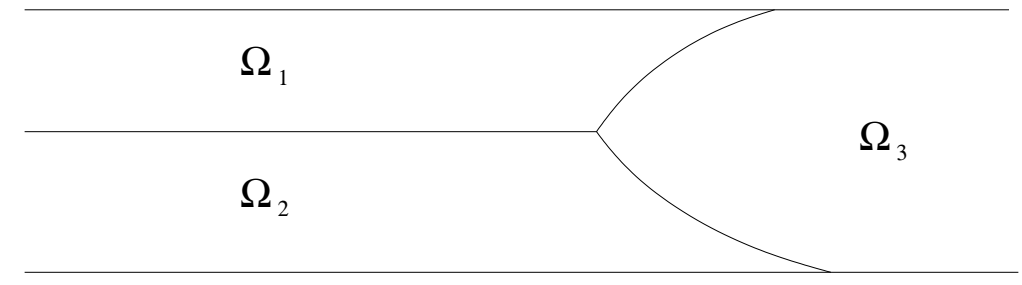

Figure 3. The new decomposition satisfies (1.6)-(1.7).

The main result is the following. Here we denote by $H^{\infty}$ the set of smooth functions with bounded $H^{s}$-norms for any positive integer $s$.

Theorem 1.1. Let (1.2)-(1.9) be assumed and let $a_{i j}, b_{i}, c, K$ be $H^{\infty}$ in $\mathbb{R}^{n} \times[0, T]$. Then for any $H^{\infty}$ functions $w_{0}, \tilde{w}_{0}$ in $\mathbb{R}^{n}$ and $f$ in $\mathbb{R}^{n} \times[0, T]$, there is an $H^{\infty}$ solution $w$ of equation (1.1) with the condition

$$
w(x, 0)=w_{0}(x), \quad \partial_{t} w(x, 0)=\tilde{w}_{0}(x) .
$$

Moreover, there exists a positive constant $N$, depending only on the decomposition in (1.4), such that the following estimate holds for any integer $s \geq 1$ :

$$
\|w\|_{s} \leq c_{s}\left(\left\|w_{0}\right\|_{s+N+1}+\left\|\tilde{w}_{0}\right\|_{s+N}+\|f\|_{s+N}\right)
$$

where the positive constant $c_{s}$ depends only on $s, \lambda, \Lambda, T, C^{s+N}$-norms of $a_{i j}, b_{i}$, $c$ and $K$, and the decomposition in (1.4). 
By the property of the propagation at finite speed of solutions to hyperbolic equations, Theorem 1.1 implies that the Cauchy problem of (1.1) always admits a smooth solution provided that $w_{0}$ and $\tilde{w}_{0}$ are smooth.

As (1.10) shows, there is a loss of derivatives in the solution. This is due to the degeneracy of the hyperbolic equation. The loss is related to how many times $\partial_{t} K$ changes its sign. The integer $N$ will be specified in section 5 . In both Figure 1 and Figure $3, N=2$.

In the special case when $K$ does not depend on the spatial variables, i.e., $K=$ $K(t)$, (1.4)-(1.9) are reduced to the following requirement:

$$
\partial_{t} K \text { changes its sign finitely many times in }(0, T) \text {. }
$$

Specifically, we require for some integer $N$

$$
\begin{aligned}
& {[0, T]=\bigcup_{l=0}^{N}\left[t_{l}^{-}, t_{l}^{+}\right],} \\
& \partial_{t} K \geq 0 \text { or } \leq 0 \text { in }\left[t_{l}^{-}, t_{l}^{+}\right] \text {for each } l,
\end{aligned}
$$

where $0=t_{N}^{-}<t_{N}^{+}=t_{N-1}^{-}<\cdots<t_{0}^{-}<t_{0}^{+}=T$. This is because in the decomposition (1.4), we have instead of (1.6)

$$
\Omega_{l}=\mathbb{R}^{n} \times\left(t_{l}^{-}, t_{l}^{+}\right),
$$

and hence its boundary is given by constant functions. Therefore, (1.8) and (1.9) are automatically satisfied.

Corollary 1.2. Let (1.2)-(1.3) be assumed, and let $a_{i j}, b_{i}, c$ be $H^{\infty}$ in $\mathbb{R}^{n} \times[0, T]$ and $K=K(t)$ be $H^{\infty}$ in $[0, T]$. Suppose $\partial_{t} K$ changes its sign $N$ times in $(0, T)$. Then for any $H^{\infty}$ functions $w_{0}, \tilde{w}_{0}$ in $\mathbb{R}^{n}$ and $f$ in $\mathbb{R}^{n} \times(0, T)$, there is an $H^{\infty}$ solution $w$ of equation (1.1) with the condition

$$
w(x, 0)=w_{0}(x), \quad \partial_{t} w(x, 0)=\tilde{w}_{0}(x) .
$$

Moreover, there holds for any integer $s \geq 1$

$$
\|w\|_{s} \leq c_{s}\left(\left\|w_{0}\right\|_{s+N+1}+\left\|\tilde{w}_{0}\right\|_{s+N}+\|f\|_{s+N}\right),
$$

where the positive constant $c_{s}$ depends only on $s, N, \lambda, \Lambda, T$ and $C^{s+N}$-norms of $a_{i j}, b_{i} c$ and $K$.

Note the integer $N$ has a simple characterization in Corollary 1.2

Now we describe the method of proof. An essential step is to transform the hyperbolic equation to a special symmetric hyperbolic system. The symmetry is important in the discussion in the differential system, as shown in [4] and [5]. In fact, the method of transform is due to Friedrichs in 5 . The new differential system has a special feature that the expression $\partial_{t} K$ appears in a significant position. Such a system allows us to treat the cases $\partial_{t} K \geq 0$ and $\partial_{t} K \leq 0$ separately and derive a priori estimates when $\partial_{t} K$ has a fixed sign. In the estimates, the loss of derivatives occurs. With the estimates for the differential system, we can obtain estimates for solutions of the hyperbolic equation in the region where $\partial_{t} K$ has a fixed sign. Last, we put all those regions together and get an estimate for the solution of the Cauchy problem.

Our method is elementary in the sense that it is based on the standard energy estimates. We avoid the micro local analysis, a standard technique in treating 
degenerate hyperbolic equations. (See [7, [10 and [11.) Our method can be modified to treat the nonlinear equation

$$
\partial_{t t} w-K(x, t) \sum_{i, j=1}^{n} a_{i j}(x, t) \partial_{i j} w-b\left(x, t, w, \partial_{x} w\right)=0 \quad \text { in } \mathbb{R}^{n} \times(0, T),
$$

where $K$ and $a_{i j}$ are as before and $b$ is a smooth function in its arguments satisfying for any function $w$ and any $i=1, \cdots, n$,

$$
\left|\partial_{w_{i}} b\left(\cdot, \cdot, w, \partial_{x} w\right)\right| \leq c\left(|w|_{C^{1}}\right) \sqrt{K}, \quad \text { in } \mathbb{R}^{n} \times(0, T) .
$$

To study (1.11), we need more precise estimates for solutions of linear equations and to keep track of the dependence on the coefficients. Since the loss of derivatives occurs, we need to employ the Nash-Moser iteration to prove the existence of solutions of (1.11). In order to present the idea clearly, we shall focus on the linear equation (1.1) and not pursue the solvability of the nonlinear equation (1.11).

In 6], we used a similar idea to discuss the local isometric embedding of surfaces with nonpositive Gauss curvature. There, we need to find a solution of a nonlinear degenerate hyperbolic system in a neighborhood of the origin in $(x, t) \in \mathbb{R} \times \mathbb{R}$.

The paper is organized in the following way. In section 2 , we shall change the hyperbolic equation into a special symmetric hyperbolic system. Then in the following section, we shall derive a priori estimates for solutions of this differential system. In section 4, we shall derive a priori estimates for solutions of the hyperbolic equation in the domain where $\partial_{t} K$ has a fixed sign. Then in section 5 , we shall put those estimates together and derive an estimate for the Cauchy problem. With such an estimate, we shall prove the existence of solutions of the degenerate hyperbolic equation by the regularization.

\section{Derivation of AN EQUivalent DifFerential SYSTEM}

In this section, we shall change the hyperbolic equation to a differential system which will play an important role in the later discussion.

We consider the equation

$$
\partial_{t t} w-k^{2}(x, t) \sum_{i, j=1}^{n} a_{i j}(x, t) \partial_{i j} w-\sum_{i=1}^{n} b_{i}(x, t) \partial_{i} w-c(x, t) w=f,
$$

where $\left(a_{i j}\right)$ is positive definite and $k$ is positive in $\mathbb{R}^{n} \times[0, T]$. Here we assume $\left(a_{i j}\right)$, $b_{i}, c$ and $k^{2}$ are smooth and $k$ is Lipschitz, with

$$
\lambda I \leq\left(a_{i j}\right) \leq \Lambda I \quad \text { in } \mathbb{R}^{n} \times[0, T]
$$

and

$$
0<k \leq k_{0} \quad \text { in } \mathbb{R}^{n} \times[0, T]
$$

for some positive constants $\lambda \leq \Lambda$ and $k_{0}$. For convenience, we write

$$
A=\left(a_{i j}\right)
$$

For later reference, we denote for $i=1, \cdots, n$

$$
\mathbf{a}_{\mathbf{i}}=\left(a_{i 1}, \cdots, a_{i n}\right)
$$

and

$$
\mathbf{b}=\left(b_{1}, \cdots, b_{n}\right)
$$


We also assume

$$
|\mathbf{b}| \leq C k \quad \text { in } \mathbb{R}^{n} \times[0, T]
$$

for some positive constant $C$.

Set

$$
U \equiv\left(\begin{array}{c}
u_{-1} \\
u_{0} \\
u_{1} \\
\vdots \\
u_{n}
\end{array}\right)=\left(\begin{array}{c}
w \\
\partial_{t} w \\
\partial_{1} w \\
\vdots \\
\partial_{n} w
\end{array}\right) .
$$

This is an $(n+2)$-vector. We write equation (2.1) as

$$
\begin{aligned}
\partial_{t} u_{-1} & =u_{0} \\
\partial_{t} u_{0} & =k^{2} \sum_{i, j=1}^{n} a_{i j} \partial_{i} u_{j}+\sum_{i=1}^{n} b_{i} u_{i}+c u_{-1}+f, \\
\sum_{j=1}^{n} k^{2} a_{i j} \partial_{t} u_{j} & =\sum_{j=1}^{n} k^{2} a_{i j} \partial_{j} u_{0}, \quad i=1, \cdots, n .
\end{aligned}
$$

Then we put (2.6) into the matrix form

$$
\tilde{A}_{0} \partial_{t} U=\sum_{i=1}^{n} \tilde{A}_{i} \partial_{i} U+\tilde{B} U+\tilde{F}
$$

where

$$
\begin{aligned}
\tilde{A}_{0} & =\left(\begin{array}{cc}
I_{2 \times 2} & \\
& k^{2} A
\end{array}\right), \\
\tilde{A}_{i} & =k^{2}\left(\begin{array}{ccc}
0 & 0 & \mathbf{0} \\
0 & 0 & \mathbf{a}_{\mathbf{i}} \\
\mathbf{0} & \mathbf{a}_{\mathbf{i}}^{T} & 0_{n \times n}
\end{array}\right), \quad i=1, \cdots, n, \\
\tilde{B} & =\left(\begin{array}{ccc}
0 & 1 & \mathbf{0} \\
c & 0 & \mathbf{b} \\
\mathbf{0} & \mathbf{0} & 0_{n \times n}
\end{array}\right),
\end{aligned}
$$

and

$$
\tilde{F}^{T}=\left(\begin{array}{lllll}
0 & f & 0 & \cdots & 0
\end{array}\right) .
$$

Consider the transformation

$$
U=M V
$$

where the $(n+2) \times(n+2)$ matrix $M$ is given by

$$
M=\left(\begin{array}{cc}
I_{2 \times 2} & \\
& \frac{1}{k} I_{n \times n}
\end{array}\right) .
$$

Obviously $M$ is invertible and

$$
V=M^{-1} U=\left(\begin{array}{cc}
I_{2 \times 2} & \\
& k I_{n \times n}
\end{array}\right) U .
$$


Now we derive the system for $V$. First, we have by (2.7) and (2.11)

$$
\begin{aligned}
M \tilde{A}_{0} M \partial_{t} V= & \sum_{i=1}^{n} M \tilde{A}_{i} M \partial_{i} V-M \tilde{A}_{0} \partial_{t} M V \\
& +\sum_{i=1}^{n} M \tilde{A}_{i} \partial_{i} M V+M \tilde{B} M V+M \tilde{F} .
\end{aligned}
$$

We put the system (2.14) into the form

$$
A_{0} \partial_{t} V=k \sum_{i=1}^{n} A_{i} \partial_{i} V+\partial_{t} \ln k B_{0} V+B V+F,
$$

where

$$
\begin{aligned}
A_{0} & =M \tilde{A}_{0} M, \\
k A_{i} & =M \tilde{A}_{i} M, \quad i=1, \cdots, n, \\
\partial_{t} \ln k B_{0} & =-M \tilde{A}_{0} \partial_{t} M, \\
B & =\sum_{i=1}^{n} M \tilde{A}_{i} \partial_{i} M+M \tilde{B} M, \\
F & =M \tilde{F} .
\end{aligned}
$$

In the coefficient matrix for $V$, we single out the term corresponding to $\partial_{t} \ln k$ since it is not controlled. The matrix $B$ can further be written as

$$
B=\sum_{i=1}^{n} \partial_{i} k B_{i}+D_{0}
$$

where

$$
\begin{aligned}
\sum_{i=1}^{n} \partial_{i} k B_{i} & =\sum_{i=1}^{n} M \tilde{A}_{i} \partial_{i} M, \\
D_{0} & =M \tilde{B} M .
\end{aligned}
$$

By a straightforward calculation, we obtain

$$
\begin{aligned}
A_{0} & =\left(\begin{array}{cc}
I_{2} & 0 \\
0 & A
\end{array}\right), \\
A_{i} & =\left(\begin{array}{ccc}
0 & 0 & \mathbf{0} \\
0 & 0 & \mathbf{a}_{\mathbf{i}} \\
\mathbf{0} & \mathbf{a}_{\mathbf{i}}^{T} & 0_{n \times n}
\end{array}\right), \quad i=1, \cdots, n, \\
B_{0} & =\left(\begin{array}{cc}
0_{2} & \\
& A
\end{array}\right), \\
B_{i} & =-\left(\begin{array}{ccc}
0 & 0 & 0 \\
0 & 0 & \mathbf{a}_{\mathbf{i}} \\
& 0_{n \times(n+2)} & 0
\end{array}\right), \quad i=1, \cdots, n, \\
D_{0} & =\left(\begin{array}{ccc}
0 & 1 & \mathbf{0} \\
c & 0 & \frac{1}{k} \mathbf{b} \\
& 0 & 0_{n \times(n+2)}
\end{array}\right),
\end{aligned}
$$


and

$$
F^{T}=\left(\begin{array}{lllll}
0 & f & 0 & \cdots & 0
\end{array}\right) .
$$

Note that the matrices $A_{0}, A_{1}, \cdots, A_{n}, B_{0}, B_{1}, \cdots, B_{n}$ depend only on the matrix $\left(a_{i j}\right)$, independent of $k$, and the matrix $D_{0}$ depends only on $b_{i}, c$ and $k$.

For future reference, we derive the differential system for

$$
W=\frac{V}{k} \text {. }
$$

A direct calculation yields

$$
\begin{aligned}
A_{0} \partial_{t} W= & k \sum_{i=1}^{n} A_{i} \partial_{i} W-\partial_{t} \ln k\left(A_{0}-B_{0}\right) W \\
& +\left(\sum_{i=1}^{n} \partial_{i} k A_{i}+B\right) W+\frac{F}{k} .
\end{aligned}
$$

Next we state some properties of the coefficient matrices for (2.15) and (2.23).

Lemma 2.1. The matrices $A_{0}, A_{1}, \cdots, A_{n}$ are symmetric in $\mathbb{R}^{n} \times[0, T]$.

Proof. This is obvious by (2.18) and (2.19).

Lemma 2.2. The matrices $B_{0}$ and $A_{0}-B_{0}$ are semi-positive definite.

Proof. This is obvious by (2.18) and (2.20).

Lemma 2.3. If $k$ is Lipschitz, then there holds

$$
|B|_{L^{\infty}} \leq C\left(1+\left|a_{i j}\right|_{L^{\infty}}+|c|_{L^{\infty}}+\left|\frac{\mathbf{b}}{k}\right|_{L^{\infty}}\right),
$$

where $C$ is a positive constant depending only on the Lipschitz norms of $k$. Moreover, for any $(n+2)$-vector $Z$ with $Z^{T}=\left(z_{1}, z_{2}, 0, \cdots, 0\right)$, there holds

$$
B Z=\left(z_{2}, c z_{1}, 0, \cdots, 0\right) .
$$

Proof. By (2.17) and the expressions for $B_{1}, \cdots, B_{n}$ and $D_{0}$, (2.24) follows easily. For (2.25), we note that the first two columns in the matrices $B_{1}, \cdots, B_{n}$ are zero. Hence we have $B Z=D_{0} Z$, which yields (2.25).

We also need the following relation between $V$ and $(w, \partial w)$.

Lemma 2.4. There holds

$$
|V|^{2}=|w|^{2}+\left|\partial_{t} w\right|^{2}+k^{2}\left|\partial_{x} w\right|^{2} .
$$

Proof. This follows easily by (2.13).

Next, we prove a result concerning the matrices $A_{0}, A_{1}, \cdots, A_{n}$.

Lemma 2.5. For any $\eta_{1}, \cdots, \eta_{n} \in \mathbb{R}$, if

$$
1-k\left(\sum_{i, j=1}^{n} a_{i j} \eta_{i} \eta_{j}\right)^{\frac{1}{2}}>0,
$$

then the $(n+2) \times(n+2)$ matrix

$$
A_{0}-k \sum_{i=1}^{n} \eta_{i} A_{i}
$$


is positive definite. Moreover, there holds

$$
c_{1}\left(1-k\left(\sum_{i, j=1}^{n} a_{i j} \eta_{i} \eta_{j}\right)^{\frac{1}{2}}\right) I_{n+2} \leq A_{0}-k \sum_{i=1}^{n} \eta_{i} A_{i} \leq c_{2}\left(1+k\left(\sum_{i, j=1}^{n} a_{i j} \eta_{i} \eta_{j}\right)^{\frac{1}{2}}\right) I_{n+2},
$$

where $c_{1}$ and $c_{2}$ are two positive constants depending only on $\lambda$ and $\Lambda$.

Proof. It is not difficult to calculate the characteristic polynomial of the matrix in (2.27). However, it is difficult to analyze the roots of this polynomial, even if $A=\left(a_{i j}\right)$ is diagonal. So we shall change the matrix in (2.27) to one which is easy to analyze. Consider a positive definite square root $\sqrt{A}_{0}$ of the matrix $A_{0}$ and set

$$
\left(\sqrt{A}_{0}\right)^{-1}=\left(\begin{array}{c}
\mathbf{p}_{\mathbf{1}} \\
\vdots \\
\mathbf{p}_{\mathbf{n}}
\end{array}\right) \text {. }
$$

Then we write

$$
A_{0}-k \sum_{i=1}^{n} \eta_{i} A_{i}=\sqrt{A_{0}}\left(I-k \sum_{i=1}^{n} \eta_{i}\left(\sqrt{A_{0}}\right)^{-1} A_{i}\left(\sqrt{A_{0}}\right)^{-1}\right) \sqrt{A_{0}} .
$$

Since the eigenvalues of $\sqrt{A_{0}}$ are between $\min \{1, \sqrt{\lambda}\}$ and $\max \{1, \sqrt{\Lambda}\}$, it suffices to calculate the eigenvalues of the matrix

$$
I-k \sum_{i=1}^{n} \eta_{i}\left(\sqrt{A_{0}}\right)^{-1} A_{i}\left(\sqrt{A_{0}}\right)^{-1}
$$

A straightforward calculation shows

$$
\left(\sqrt{A_{0}}\right)^{-1} A_{i}\left(\sqrt{A_{0}}\right)^{-1}=\left(\begin{array}{ccccc}
0 & 0 & 0 & \cdots & 0 \\
0 & 0 & \mathbf{a}_{\mathbf{i}} \cdot \mathbf{p}_{\mathbf{1}} & \cdots & \mathbf{a}_{\mathbf{i}} \cdot \mathbf{p}_{\mathbf{n}} \\
0 & \mathbf{a}_{\mathbf{i}} \cdot \mathbf{p}_{\mathbf{1}} & & & \\
\vdots & \vdots & & 0_{n \times n} & \\
0 & \mathbf{a}_{\mathbf{i}} \cdot \mathbf{p}_{\mathbf{n}} & & &
\end{array}\right), \quad i=1, \cdots, n .
$$

Setting

$$
d_{j}=k \sum_{i=1} \eta_{i} \mathbf{a}_{\mathbf{i}} \cdot \mathbf{p}_{\mathbf{j}}, \quad j=1, \cdots, n,
$$

then the matrix in (2.28) can be expressed as

$$
\left(\begin{array}{ccccc}
1 & 0 & 0 & \cdots & 0 \\
0 & 1 & -d_{1} & \cdots & -d_{n} \\
0 & -d_{1} & & & \\
\vdots & \vdots & & I_{n \times n} & \\
0 & -d_{n} & & &
\end{array}\right)
$$

Its characteristic polynomial is given by

$$
(\lambda-1)^{n}\left((\lambda-1)^{2}-\sum_{j=1}^{n}\left|d_{j}\right|^{2}\right)
$$


and hence its eigenvalues are given by

$$
1-\left(\sum_{j=1}^{n}\left|d_{j}\right|^{2}\right)^{\frac{1}{2}}, 1, \cdots, 1(n \text { times }), 1+\left(\sum_{j=1}^{n}\left|d_{j}\right|^{2}\right)^{\frac{1}{2}} .
$$

By (2.29), we can verify

$$
\sum_{j=1}^{n}\left|d_{j}\right|^{2}=k^{2} \sum_{i, j=1}^{n} a_{i j} \eta_{i} \eta_{j}
$$

This finishes the proof.

In the rest of the section, we shall consider the equations of the $x$-derivatives of $w$. Fix an integer $s \geq 1$. For any multi-index $\gamma \in \mathbb{Z}_{+}^{n}$ with $|\gamma|=s$, apply $\partial^{\gamma}=\partial_{x}^{\gamma}$ to both sides of (2.1) to get

$$
\begin{gathered}
\partial_{t t} \partial^{\gamma} w-k^{2} \sum_{i, j=1}^{n} a_{i j} \partial_{i j} \partial^{\gamma} w-\sum_{i=1}^{n} b_{i}(x, t) \partial_{i} \partial^{\gamma} w-c \partial^{\gamma} w \\
-\sum_{i, j=1}^{n} \sum_{\eta+\eta^{\prime}=\gamma,|\eta|=1,2} \partial^{\eta}\left(k^{2} a_{i j}\right) \partial^{\eta^{\prime}+\mathbf{e}_{\mathbf{i}}+\mathbf{e}_{\mathbf{j}} w} \\
-\sum_{i=1}^{n} \sum_{\eta+\eta^{\prime}=\gamma,|\eta|=1} \partial^{\eta} b_{i} \partial^{\eta^{\prime}+\mathbf{e}_{\mathbf{i}}} w=f_{\gamma},
\end{gathered}
$$

where

$$
\begin{aligned}
f_{\gamma}= & \partial^{\gamma} f+\sum_{\eta+\eta^{\prime}=\gamma,|\eta| \geq 3} \partial^{\eta}\left(k^{2} a_{i j}\right) \partial^{\eta^{\prime}+\mathbf{e}_{\mathbf{i}}+\mathbf{e}_{\mathbf{j}}} w \\
& +\sum_{\eta+\eta^{\prime}=\gamma,|\eta| \geq 2} \partial^{\eta} b_{i} \partial^{\eta^{\prime}+\mathbf{e}_{\mathbf{i}}} w+\sum_{\eta+\eta^{\prime}=\gamma,|\eta| \geq 1} \partial^{\eta} c \partial^{\eta^{\prime}} w .
\end{aligned}
$$

Here $f_{\gamma}$ is the linear combination of $\partial^{\gamma} f, w, \partial_{x} w, \cdots, \partial_{x}^{s-1} w$ with coefficients given by $1, k^{2} a_{i j}, b_{i}, c$ and their derivatives up to order $s$. Note that the fifth and sixth terms in the left side of (2.30) consist of $x$-derivatives of $w$ of the order $s$ and $s+1$ and may not be written as a linear combination of $\partial^{\gamma} w$ and $\partial_{i} \partial^{\gamma} w, i=1, \cdots, n$, for the fixed $\gamma$. Hence we need to consider all $\gamma$ with $|\gamma|=s$.

Let $W_{s}$ be the column vector with entries given by all $x$-derivatives of $w$ of the order $s$, i.e.,

$$
W_{s}^{T}=\left(\begin{array}{llll}
\partial_{1}^{s} w & \partial_{1}^{s-1} \partial_{2} w & \cdots & \partial_{n}^{s} w
\end{array}\right) .
$$

This is a vector of $m$ components for some positive integer $m=m(s)$. Now we form a huge differential system by putting together the equations corresponding to all $\gamma$ with $|\gamma|=s$ in (2.30). We can write this system in the form

$$
\partial_{t t} W_{s}-k^{2} \sum_{i, j=1}^{n} a_{i j} \partial_{i j} W_{s}-\sum_{i=1}^{n} B_{s i} \partial_{i} W_{s}-C_{s} W_{s}=F_{s},
$$

where $B_{s 1}, \cdots, B_{s n}, C_{s}$ are $m \times m$ matrices satisfying

$$
\left|B_{s i}\right| \leq C k,
$$

and $F_{s}$ is an $m$-vector given by

$$
F_{s}^{T}=\left(f_{(s, 0, \cdots, 0)} \quad f_{(s-1,1,0, \cdots, 0)} \quad \cdots \quad f_{(0, \cdots, 0, s)}\right) .
$$


Now introduce

$$
U^{(s)} \equiv\left(\begin{array}{c}
U_{-1}^{(s)} \\
U_{0}^{(s)} \\
U_{1}^{(s)} \\
\vdots \\
U_{n}^{(s)}
\end{array}\right)=\left(\begin{array}{c}
W_{s} \\
\partial_{t} W_{s} \\
\partial_{1} W_{s} \\
\vdots \\
\partial_{n} W_{s}
\end{array}\right) .
$$

This is an $m(n+2)$-vector. Then $U^{(s)}$ satisfies

$$
\begin{aligned}
\partial_{t} U_{-1}^{(s)} & =U_{0}^{(s)} \\
\partial_{t} U_{0}^{(s)} & =k^{2} \sum_{i, j=1}^{n} a_{i j} \partial_{i} U_{j}^{(s)}+\sum_{i=1}^{n} B_{s i} U_{i}^{(s)}+C_{s} U_{-1}^{(s)}+F_{s}, \\
\sum_{j=1} k^{2} a_{i j} \partial_{t} U_{j}^{(s)} & =\sum_{j=1} k^{2} a_{i j} \partial_{j} U_{0}^{(s)}, \quad i=1, \cdots, n .
\end{aligned}
$$

This is a system of $m(n+2)$ equations. Compare (2.36) with (2.6). The matrix form of (2.36) is

$$
\tilde{A}_{0}^{(s)} \partial_{t} U^{(s)}=\sum_{i=1}^{n} \tilde{A}_{i}^{(s)} \partial_{i} U^{(s)}+\tilde{B}_{(s)} U^{(s)}+\tilde{F}_{(s)},
$$

where $\tilde{A}_{i}^{(s)}, i=0, \cdots, n$, and $\tilde{B}_{(s)}$ are some $m(n+2) \times m(n+2)$ matrices and $\tilde{F}_{(s)}$ is an $m(n+2)$-vector given by

$$
\tilde{F}_{(s)}=\left(\begin{array}{c}
0 \\
F_{s} \\
\vdots \\
0
\end{array}\right) .
$$

The matrix $\tilde{A}_{l}^{(s)}, l=0,1, \cdots, n$, in (2.37) is obtained as follows. In $\tilde{A}_{l}=\left(\tilde{a}_{i j}^{l}\right)$ in (2.8) and (2.9), we replace each scalar entry $\tilde{a}_{i j}^{l}$ by $\tilde{a}_{i j}^{l} I_{m \times m}$ to get an $m(n+2) \times$ $m(n+2)$ matrix $\tilde{A}_{l}^{(s)}$. We should do the same thing for $M$ in (2.12), $A_{i}$ in (2.18) and (2.19) and $B_{0}$ in (2.20) to get $M^{(s)}$ and $A_{i}^{(s)}, i=0,1, \cdots, n$, and $B_{0}^{(s)}$. Now we introduce

$$
U^{(s)}=M^{(s)} V^{(s)} .
$$

Then by a similar calculation, we find that $V^{(s)}$ satisfies

$$
A_{0}^{(s)} \partial_{t} V^{(s)}=k \sum_{i=1}^{n} A_{i}^{(s)} \partial_{i} V^{(s)}+\partial_{t} \ln k B_{0}^{(s)} V^{(s)}+B_{(s)} V^{(s)}+F^{(s)},
$$

where $B_{(s)}$ is some $m(n+2) \times m(n+2)$ matrix and

$$
F^{(s)}=\left(\begin{array}{c}
0 \\
F_{s} \\
0 \\
\vdots \\
0
\end{array}\right) .
$$


Note that $A_{0}^{(s)}, A_{1}^{(s)}, \cdots, A_{n}^{(s)}$ are symmetric and that $B_{0}^{(s)}$ and $A_{0}^{(s)}-B_{0}^{(s)}$ are semi-positive definite. Hence $A_{0}^{(s)}, A_{1}^{(s)}, \cdots, A_{n}^{(s)}$ and $B_{0}^{(s)}$ satisfy Lemma 2.1 and Lemma 2.2. Moreover, the matrix $B_{(s)}$ satisfies the obviously-modified Lemma 2.3. Appropriate forms of Lemma 2.4 and 2.5] also hold in the present setting.

\section{A PRIORI ESTIMATES FOR THE DIFFERENTIAL SYSTEM IN A SPECIAL DOMAIN}

In this section, we shall establish a priori estimates for solutions of the differential system derived in the previous section.

We consider the differential system for an $m$-vector $V$ in $\mathbb{R}^{n} \times(0, T)$

$$
A_{0} \partial_{t} V-k \sum_{i=1}^{n} A_{i} \partial_{i} V=\partial_{t} \ln k B_{0} V+B V+F,
$$

where $k$ is a Lipschitz function with $0<k \leq k_{0}$ in $\mathbb{R}^{n} \times(0, T)$ and $A_{0}, A_{1}, \cdots, A_{n}$, $B_{0}, B$ are $m \times m$ function-valued matrices in $\mathbb{R}^{n} \times(0, T)$ with $A_{i} \in$ Lip, $i=$ $0,1, \cdots, n$, and $B_{0}, B \in L^{\infty}$. Moreover, we assume

$$
A_{0}, A_{1}, \cdots, A_{n} \text { are symmetric }
$$

and

$$
B_{0} \text { and } A_{0}-B_{0} \text { are semi-positive definite in } \mathbb{R}^{n} \times(0, T) \text {. }
$$

Let $D \subset \mathbb{R}^{n}$ be a domain with a piecewise smooth boundary. Here $D$ may be unbounded. Let $\Sigma_{ \pm}$be two surfaces over $D$ defined by piecewise smooth functions $t=t_{ \pm}(x)$ with

$$
\begin{aligned}
& 0 \leq t_{-}(x)<t_{+}(x) \leq T \text { for } x \in D, \\
& t_{-}(x)=t_{+}(x) \text { for } x \in \partial D
\end{aligned}
$$

and

$$
\left|\partial t_{ \pm}(x)\right| \leq \eta_{0} \text { for } x \in D
$$

for some positive constant $\eta_{0}$. Set

$$
\Omega=\left\{(x, t) ; x \in D, t_{-}(x)<t<t_{+}(x)\right\} .
$$

Throughout the section, we always assume $\Sigma_{ \pm}$is space-like, i.e.,

$$
A_{0}-k \sum_{i=1}^{n} \partial_{i} t_{ \pm} A_{i} \quad \text { is positive definite on } \Sigma_{ \pm} \text {. }
$$

Moreover, we assume

$$
\eta_{1} I \leq \frac{1}{\sqrt{1+\left|\nabla t_{ \pm}\right|^{2}}}\left(A_{0}-k \sum_{i=1}^{n} \partial_{i} t_{ \pm} A_{i}\right) \leq \eta_{2} I \quad \text { on } \Sigma_{ \pm},
$$

for some positive constants $\eta_{1}$ and $\eta_{2}$.

In the following two results, we always assume $V$ is identically zero for large $|x|$ if $D$ is unbounded.

Lemma 3.1. Let (3.2)-(3.7) be assumed and let $V \in C^{1}(\bar{\Omega})$ be a solution of (3.1). If $\partial_{t} k \leq 0$ in $\Omega$, then there holds for any $\mu \geq \mu_{0}$

$$
\int_{\Sigma_{+}} e^{-\mu t}|V|^{2}+\int_{\Omega} e^{-\mu t}|V|^{2} \leq c_{0}\left\{\int_{\Sigma_{-}} e^{-\mu t}|V|^{2}+\int_{\Omega} e^{-\mu t}|F|^{2}\right\},
$$


where $\mu_{0}$ and $c_{0}$ are positive constants depending only on $\eta_{1}, \eta_{2},|B|_{L^{\infty}},|k|_{\text {Lip }}$ and $\left|A_{i}\right|_{\text {Lip }}, i=0,1, \cdots, n$.

Proof. Taking the inner product of $2 e^{-\mu t} V$ and both sides of (3.1) for $\mu>0$ and integrating by parts over the domain $\Omega$, we get

$$
\begin{aligned}
& \int_{\Sigma_{+}} \frac{1}{\sqrt{1+\left|\nabla t_{+}\right|^{2}}} e^{-\mu t} V^{T}\left(A_{0}-k \sum_{i=1}^{n} \partial_{i} t_{+} A_{i}\right) V \\
& \quad+\mu \int_{\Omega} e^{-\mu t}|V|^{2}-\int_{\Omega} e^{-\mu t} \partial_{t} \ln k V^{T} B_{0} V \\
& =\int_{\Sigma_{-}} \frac{1}{\sqrt{1+\left|\nabla t_{-}\right|^{2}}} e^{-\mu t} V^{T}\left(A_{0}-k \sum_{i=1}^{n} \partial_{i} t_{-} A_{i}\right) V \\
& \quad+\int_{\Omega} e^{-\mu t} V^{T}\left(2 B+\partial_{t} A_{0}-\sum_{i=1}^{n} \partial_{i}\left(k A_{i}\right)\right) V+2 \int_{\Omega} e^{-\mu t} V^{T} F .
\end{aligned}
$$

In view of the hypothesis $\partial_{t} k \leq 0$, the third term in the left-hand side of (3.9) is nonnegative. Moreover, the second term in the right-hand side of (3.9) is controlled by

$$
C \int_{\Omega} e^{-\mu t}|V|^{2}
$$

By noting the definition of $\eta_{1}$ and $\eta_{2}$ in (3.7), we obtain

$$
\begin{aligned}
\eta_{1} \int_{\Sigma_{+}} e^{-\mu t}|V|^{2} & +\mu \int_{\Omega} e^{-\mu t}|V|^{2} \leq \eta_{2} \int_{\Sigma_{-}} e^{-\mu t}|V|^{2} \\
& +c_{0}^{\prime} \int_{\Omega} e^{-\mu t}|V|^{2}+\int_{\Omega} e^{-\mu t}|F|^{2}
\end{aligned}
$$

where $c_{0}^{\prime}$ is a positive constant independent of $\mu$. Thus (3.8) is proved by choosing $\mu$ sufficiently large.

Lemma 3.2. Let (3.2)-(3.7) be assumed and let $V \in C^{1}(\bar{\Omega})$ be a solution of (3.1). If $\partial_{t} k \geq 0$ in $\Omega$, then there holds for any $\mu \geq \mu_{0}$

$$
\int_{\Sigma_{+}} e^{-\mu t}\left|\frac{V}{k}\right|^{2}+\int_{\Omega} e^{-\mu t}\left|\frac{V}{k}\right|^{2} \leq c_{0}\left\{\int_{\Sigma_{-}} e^{-\mu t}\left|\frac{V}{k}\right|^{2}+\int_{\Omega} e^{-\mu t}\left|\frac{F}{k}\right|^{2}\right\},
$$

where $\mu_{0}$ and $c_{0}$ are positive constants depending only on $\eta_{1}, \eta_{2},|B|_{L^{\infty}},|k|_{\text {Lip }}$ and $\left|A_{i}\right|_{\text {Lip }}, i=0,1, \cdots, n$.

Proof. By (3.1), $W=V / k$ satisfies the differential system

$$
A_{0} \partial_{t} W=k \sum_{i=1}^{n} A_{i} \partial_{i} W-\partial_{t} \ln k\left(A_{0}-B_{0}\right) W+\left(\sum_{i=1}^{n} \partial_{i} k A_{i}+B\right) W+\frac{F}{k}
$$

Then we may proceed as in the proof of Lemma 3.1 to get (3.10). We omit the details.

Remark. For later application, we shall take a fixed $\mu \geq \mu_{0}$. In the present paper, we only treat the case in which $T$ is finite. In this case, it is not necessary to have $e^{-\mu t}$ present in the integrals later on. We only need to require that $c_{0}$ depends on $T$ additionally. However, if $T$ is infinite, it is essential to have $e^{-\mu t}$ present in all the integrals. 


\section{A PRiori estimates For the HYPERBoliC EQUATION IN A SPECIAL DOMAIN}

In this section, we shall derive estimates for solutions of (2.1) in a special domain introduced in section 3 .

Throughout the section, we assume $D \subset \mathbb{R}^{n}$ and $\Omega \subset \mathbb{R}^{n} \times(0, T)$ are given in (3.4)- (3.6). We consider the equation

$$
\partial_{t t} w-k^{2}(x, t) \sum_{i, j=1}^{n} a_{i j}(x, t) \partial_{i j} w-\sum_{i=1}^{n} b_{i}(x, t) \partial_{i} w-c(x, t) w=f \text { in } \bar{\Omega} .
$$

We also assume $a_{i j}, b_{i}, c$ and $k$ satisfy (2.2), (2.3) and (2.4). Furthermore, we assume

$$
\begin{aligned}
\eta_{1} & \leq \frac{1}{\sqrt{1+\left|\partial_{x} t_{ \pm}\right|^{2}}}\left(1-k\left(\sum_{i, j=1}^{n} a_{i j} \partial_{i} t_{ \pm} \partial_{j} t_{ \pm}\right)^{\frac{1}{2}}\right) \\
& \leq \frac{1}{\sqrt{1+\left|\partial_{x} t_{ \pm}\right|^{2}}}\left(1+k\left(\sum_{i, j=1}^{n} a_{i j} \partial_{i} t_{ \pm} \partial_{j} t_{ \pm}\right)^{\frac{1}{2}}\right) \leq \eta_{2} \quad \text { on } \Sigma_{l}^{ \pm},
\end{aligned}
$$

for some positive constants $\eta_{1}$ and $\eta_{2}$.

We always assume the solution $w$ is zero for large $|x|$ if $D$ is unbounded.

For any function $w$, we set

$$
\begin{aligned}
\partial_{x} w & =\left(\partial_{1} w, \cdots, \partial_{n} w\right), \\
\partial w & =\left(\partial_{t} w, \partial_{1} w, \cdots, \partial_{n} w\right),
\end{aligned}
$$

and similar expressions for higher order derivatives. We also set for $s \geq 1$

$$
\begin{aligned}
& |w|_{s}^{2}=\sum_{|\gamma| \leq s}\left|\partial^{\gamma} w\right|^{2}, \\
& |w|_{(0, s)}^{2}=\sum_{|\gamma| \leq s}\left|\partial_{x}^{\gamma} w\right|^{2} .
\end{aligned}
$$

We adopt similar notations for vector-valued functions.

Now we derive an estimate of solutions to (4.1). The discussion in the previous sections plays an important role. Recall that $V$ introduced in (2.11) satisfies (2.15). Since the coefficient matrices in (2.15) satisfy Lemmas 2.1, 2.2, 2.3 and 2.5, then (3.2), (3.3) and (3.7) hold. In particular, (3.7) holds by Lemma 2.5, (3.5) and (4.2).

We first consider a region where $\partial_{t} k \geq 0$. We compare our solutions with the solution to a simple differential system. The difference satisfies the system studied in the previous section.

Lemma 4.1. Let $w$ be a $C^{2}(\bar{\Omega})$ solution to (4.1). Suppose $\partial_{t} k^{2} \geq 0$ in $\Omega$. Then there holds

$$
\int_{\Sigma_{+}}|w|_{1}^{2}+\int_{\Omega}|w|_{1}^{2} \leq c_{1}\left\{\int_{\Sigma_{-}}|w|_{2}^{2}+\int_{\Omega}|f|_{(0,1)}^{2}\right\}
$$

where $c_{1}$ is a positive constant depending only on $T, \lambda, \eta_{0}, \eta_{1}, \eta_{2}$, the Lipschitz norm of $k$ and $C^{1}$-norms of $a_{i j}, b_{i}, c$ and $k^{2}$. 
Proof. Consider the initial value problem

$$
\begin{aligned}
\partial_{t} z_{1}-z_{2}=0, \partial_{t} z_{2}-c z_{1} & =f \quad \text { in } \Omega, \\
z_{1}=w, z_{2} & =w_{t} \quad \text { on } \Sigma_{-} .
\end{aligned}
$$

First we have

$$
\int_{\Sigma_{+}}\left(\left|z_{1}\right|^{2}+\left|z_{2}\right|^{2}\right)+\int_{\Omega}\left(\left|z_{1}\right|^{2}+\left|z_{2}\right|^{2}\right) \leq c_{0}^{\prime}\left\{\int_{\Sigma_{-}}|w|_{1}^{2}+\int_{\Omega}|f|^{2}\right\},
$$

for some constant $c_{0}^{\prime}$ depending only on the $L^{\infty}$-norm of $c$. We obtain (4.4) by multiplying $2 e^{-\mu t} z_{1}$ and $2 e^{-\mu t} z_{2}$ to two equations in (4.3) respectively for some sufficiently large $\mu>0$ and integrating over $\Omega$ as in the proof of Lemma 3.1 We omit the details since it is similar and easier. Now we differentiate (4.3) with respect to $x_{k}, k=1, \cdots, n$, and consider the system satisfied by $\partial_{k} z_{1}$ and $\partial_{k} z_{2}$. Similarly we get

$$
\int_{\Omega}\left(\left|\partial_{x} z_{1}\right|^{2}+\left|\partial_{x} z_{2}\right|^{2}\right) \leq c_{0}^{\prime}\left\{\int_{\Sigma_{-}}|w|_{2}^{2}+\int_{\Omega}\left(|f|^{2}+\left|\partial_{x} f\right|^{2}\right)\right\} .
$$

We remark that, by (4.1) and (4.3), $\partial z_{1}$ and $\partial z_{2}$ on $\Sigma_{-}$can be expressed by a linear combination of $w$ and its derivatives up to order 2 restricted on $\Sigma_{-}$.

Recall that $V$ introduced in (2.11) satisfies (2.15). Introduce

$$
Z^{T}=\left(z_{1}, z_{2}, 0, \cdots, 0\right) .
$$

Then by (2.15), we have

$$
\begin{aligned}
A_{0} \partial_{t}(V-Z)= & k \sum_{i=1}^{n} A_{i} \partial_{i}(V-Z)+\partial_{t} \ln k B_{0}(V-Z)+B(V-Z) \\
& -\left(A_{0} \partial_{t} Z-k \sum_{i=1}^{n} A_{i} \partial_{i} Z-\partial_{t} \ln k B_{0} Z-B Z-F\right) .
\end{aligned}
$$

Note $B_{0} Z=0$ by (2.20). We also have $A_{0} \partial_{t} Z=B Z+F$ by (4.3), (2.21) and (2.25). Hence $V-Z$ satisfies

$$
\partial_{t}(V-Z)=k \sum_{i=1}^{n} A_{i} \partial_{i}(V-Z)+\partial_{t} \ln k B_{0}(V-Z)+B(V-Z)+\hat{F},
$$

with

$$
\hat{F}=k \sum_{i=1}^{n} A_{i} \partial_{i} Z
$$

By (2.5), (2.13) and (4.3), $V-Z$ satisfies on $\Sigma_{-}$

$$
V-Z=k\left(\begin{array}{c}
0 \\
0 \\
\partial_{x} w
\end{array}\right)
$$

An application of Lemma 3.2 to (4.6)-(4.7) in $\Omega$, with a fixed $\mu \geq \mu_{0}$, yields

$$
\int_{\Sigma_{+}}\left|\frac{V-Z}{k}\right|^{2}+\int_{\Omega}\left|\frac{V-Z}{k}\right|^{2} \leq c_{0}\left\{\int_{\Sigma_{-}}\left|\partial_{x} w\right|^{2}+\int_{\Omega}\left(\left|\partial_{x} z_{1}\right|^{2}+\left|\partial_{x} z_{2}\right|^{2}\right)\right\} .
$$


By Lemma 2.4, we have

$$
\begin{aligned}
\left|\frac{V-Z}{k}\right|^{2} & =\left|\frac{w-z_{1}}{k}\right|^{2}+\left|\frac{\partial_{t} w-z_{2}}{k}\right|^{2}+\left|\partial_{x} w\right|^{2} \\
& \geq C\left(\left|w-z_{1}\right|^{2}+\left|\partial_{t} w-z_{2}\right|^{2}+\left|\partial_{x} w\right|^{2}\right) .
\end{aligned}
$$

This implies

$$
\begin{gathered}
\int_{\Sigma_{+}}|w|_{1}^{2}+\int_{\Omega}|w|_{1}^{2} \leq C\left\{\int_{\Sigma_{-}}\left|\partial_{x} w\right|^{2}+\int_{\Sigma_{+}}\left(\left|z_{1}\right|^{2}+\left|z_{2}\right|^{2}\right)\right. \\
\left.+\int_{\Omega}\left(\left|z_{1}\right|^{2}+\left|z_{2}\right|^{2}+\left|\partial_{x} z_{1}\right|^{2}+\left|\partial_{x} z_{2}\right|^{2}\right)\right\} .
\end{gathered}
$$

We finish the proof by substituting (4.4) and (4.5) in (4.8).

Now we study the case when $\partial_{t} k \leq 0$. For $\partial w$, the $\partial_{t} w$ component is good and there is a degeneracy in the $\partial_{x} w$ component. In order to get an estimate on $\partial_{x} w$, we need information on $\partial_{t} w$ and its $x$ derivative.

Lemma 4.2. Let $w$ be a $C^{3}(\bar{\Omega})$ solution to (4.1). Suppose $\partial_{t} k^{2} \leq 0$ in $\Omega$. Then there holds

$$
\int_{\Sigma_{+}}|w|_{1}^{2}+\int_{\Omega}|w|_{1}^{2} \leq c_{1}\left\{\int_{\Sigma_{-}}|w|_{2}^{2}+\int_{\Omega}|f|_{(0,1)}^{2}\right\},
$$

where $c_{1}$ is a positive constant depending only on $T, \lambda, \eta_{0}, \eta_{1}, \eta_{2}$, the Lipschitz norm of $k$ and $C^{1}$-norms of $a_{i j}, b_{i}, c$ and $k^{2}$.

Proof. Recall that $V$ introduced in (2.11) satisfies (2.15). By Lemma 3.1 for a fixed $\mu \geq \mu_{0}$ and Lemma 2.4, it follows that

$$
\begin{aligned}
& \int_{\Sigma_{+}}\left(|w|^{2}+\left|\partial_{t} w\right|^{2}+\left|k \partial_{x} w\right|^{2}\right)+\int_{\Omega}\left(|w|^{2}+\left|\partial_{t} w\right|^{2}+\left|k \partial_{x} w\right|^{2}\right) \\
\leq & c_{0}\left\{\int_{\Sigma_{-}}\left(|w|^{2}+\left|\partial_{t} w\right|^{2}+\left|k \partial_{x} w\right|^{2}\right)+\int_{\Omega}|f|^{2}\right\} .
\end{aligned}
$$

This implies

$$
\int_{\Sigma_{+}}\left(|w|^{2}+\left|\partial_{t} w\right|^{2}\right)+\int_{\Omega}\left(|w|^{2}+\left|\partial_{t} w\right|^{2}\right) \leq c_{0}\left\{\int_{\Sigma_{-}}|w|_{1}^{2}+\int_{\Omega}|f|^{2}\right\} .
$$

In order to get an estimate on $\partial_{x} w$ without the weight $k$, we have to return to the original equation (4.1) and consider its $x$-derivative. To this end, consider

$$
W_{1}^{T}=\left(\begin{array}{lll}
\partial_{1} w & \cdots & \partial_{n} w
\end{array}\right)=\partial_{x} w
$$

This is a vector of $n$ components. Then by $(2.32) 1, W_{1}$ satisfies

$$
\partial_{t t} W_{1}-k^{2} \sum_{i, j=1}^{n} a_{i j} \partial_{i j} W_{1}-\sum_{i=1}^{n} B_{1 i} \partial_{i} W_{1}-C_{1} W_{1}=F_{1},
$$

where $B_{11}, \cdots, B_{1 n}, C_{1}$ are $n \times n$ matrices satisfying

$$
\left|B_{1 i}\right| \leq C k,
$$


and $F_{1}$ is the $n$-vector given by

$$
F_{1}=\left(\begin{array}{c}
\partial_{1} f+\partial_{1} c w \\
\vdots \\
\partial_{n} f+\partial_{n} c w
\end{array}\right)
$$

A similar result as (4.9) holds for $\partial_{x} w$. Hence, we have

$$
\begin{aligned}
& \int_{\Sigma_{+}}\left(\left|\partial_{x} w\right|^{2}+\left|\partial_{t x} w\right|^{2}+\left|k \partial_{x x} w\right|^{2}\right)+\int_{\Omega}\left(\left|\partial_{x} w\right|^{2}+\left|\partial_{t x} w\right|^{2}+\left|k \partial_{x x} w\right|^{2}\right) \\
\leq & c_{0}\left\{\int_{\Sigma_{-}}\left(\left|\partial_{x} w\right|^{2}+\left|\partial_{t x} w\right|^{2}+\left|k \partial_{x x} w\right|^{2}\right)+\int_{\Omega}\left|\partial_{x} f\right|^{2}+\int_{\Omega}|w|^{2}\right\} .
\end{aligned}
$$

We only need the estimate on $\partial_{x} w$, i.e.,

$$
\int_{\Sigma_{+}}\left|\partial_{x} w\right|^{2}+\int_{\Omega}\left|\partial_{x} w\right|^{2} \leq c_{0}\left\{\int_{\Sigma_{-}}\left|\partial_{x} w\right|_{1}^{2}+\int_{\Omega}\left|\partial_{x} f\right|^{2}+\int_{\Omega}|w|^{2}\right\} .
$$

Summation of (4.11) and the $\left(c_{0}+1\right)$-multiple of (4.9) yields

$$
\int_{\Sigma_{+}}|w|_{1}^{2}+\int_{\Omega}|w|_{1}^{2} \leq C\left\{\int_{\Sigma_{-}}|w|_{2}^{2}+\int_{\Omega}\left(|f|^{2}+\left|\partial_{x} f\right|^{2}\right)\right\} .
$$

This finishes the proof.

Remark. The results in Lemma 4.1 and Lemma 4.2 have similar forms. However, they are established by totally different methods. Lemma 4.1 is proved by a straightforward application of Lemma 3.2. For Lemma 4.2, we need to apply Lemma 3.1 to the system for $(w, \partial w)$ and the system for the $x$-derivatives of $(w, \partial w)$ in order to get the estimate on $\partial w$ itself. 0 .

Now we discuss higher-order derivatives of $w$. In the next result, we write $|f|_{-1}=$

Lemma 4.3. Let $s \geq 1$ be an integer and let $w$ be a $C^{s+2}(\bar{\Omega})$ solution to (4.1). Suppose $\partial_{t} k^{2} \geq 0$ or $\leq 0$ in $\Omega$. Then there holds

$$
\int_{\Sigma_{+}}|w|_{s}^{2}+\int_{\Omega}|w|_{s}^{2} \leq c_{s}\left\{\int_{\Sigma_{-}}|w|_{s+1}^{2}+\int_{\Sigma_{+}}|f|_{s-2}^{2}+\int_{\Omega}|f|_{s}^{2}\right\},
$$

where $c_{s}$ is a positive constant depending only on $s, T, \lambda, \eta_{0}, \eta_{1}, \eta_{2}$, the Lipschitz norm of $k$ and $C^{s}$-norms of $a_{i j}, b_{i}, c$ and $k^{2}$.

Proof. We shall prove (4.12) by induction. (4.12) ${ }_{1}$ is just Lemma4.1 or Lemma4.2 Assume (4.12) $j$ is true for all $j \leq s$ and $s \geq 1$. We shall prove (4.12) $s+1$. As shown in section 2, the vector $\partial_{x}^{s} w$ consisting of all the $s$-order $x$-derivatives of $w$ satisfies the differential system (2.32). It has the same structure as (4.1). An application of Lemma 4.1 or Lemma 4.2 to (2.32) in $\Omega$ yields

$$
\int_{\Sigma_{+}}\left|\partial_{x}^{s} w\right|_{1}^{2}+\int_{\Omega}\left|\partial_{x}^{s} w\right|_{1}^{2} \leq C\left\{\int_{\Sigma_{-}}\left|\partial_{x}^{s} w\right|_{2}^{2}+\sum_{|\gamma|=s} \int_{\Omega}\left|f_{\gamma}\right|_{(0,1)}^{2}\right\},
$$


where $f_{\gamma}$ is given in (2.31). Note that (2.31) implies for $|\gamma|=s$

$$
\begin{aligned}
\left|f_{\gamma}\right|_{(0,1)}^{2} & =\left|f_{\gamma}\right|^{2}+\left|\partial_{x} f_{\gamma}\right|^{2} \leq\left|\partial_{x}^{s} f\right|^{2}+\left|\partial_{x}^{s+1} f\right|^{2}+C \sum_{i=0}^{s}\left|\partial_{x}^{i} w\right|^{2} \\
& \leq|f|_{(0, s+1)}^{2}+C|w|_{(0, s)}^{2},
\end{aligned}
$$

where $C$ is a positive constant depending only on $C^{s+1}$-norms of $a_{i j}, b_{i}, c$ and $k^{2}$. Combining (4.13) and (4.14), we have

$$
\int_{\Sigma_{+}}\left|\partial_{x}^{s} w\right|_{1}^{2}+\int_{\Omega}\left|\partial_{x}^{s} w\right|_{1}^{2} \leq C\left\{\int_{\Sigma_{-}}\left|\partial_{x}^{s} w\right|_{2}^{2}+\int_{\Omega}|f|_{(0, s+1)}^{2}+\int_{\Omega}|w|_{(0, s)}^{2}\right\}
$$

It remains to evaluate the $L^{2}$-integral of $\partial_{t}^{j} \partial_{x}^{s+1-j} w$ for $2 \leq j \leq s+1$. From (4.1), it follows that

$$
\partial_{t}^{2} \partial_{x}^{s-1} w=\partial_{x}^{s-1}\left(k^{2} a_{i j} \partial_{i j} w+b_{i} \partial_{i} w+c w\right)+\partial_{x}^{s-1} f .
$$

In general, $\partial_{t}^{j} \partial_{x}^{s-j+1} w, j=2, \cdots, s+1$, is a linear combination of

$$
\partial^{s-1} f, w, \partial_{x} w, \cdots, \partial_{x}^{s+1} w, \partial_{t} w, \partial_{t} \partial_{x} w, \cdots, \partial_{t} \partial_{x}^{s} w,
$$

with coefficients given by $1, k^{2} a_{i j}, b_{i}, c$ and their derivatives up to order $s-1$. The $t$-derivative of $w$ in (4.16) is present for $j \geq 3$. Hence we obtain for $j=2, \cdots, s+1$,

$$
\left|\partial_{t}^{j} \partial_{x}^{s-j+1} w\right|^{2} \leq C\left(|f|_{s-1}^{2}+|w|_{s}^{2}+\left|\partial_{x}^{s} w\right|_{1}^{2}\right),
$$

where $C$ is a positive constant depending only on $C^{s-1}$-norms of $a_{i j}, b_{i}, c$ and $k^{2}$. Note that the last two terms in the right side have common terms. Then we have

$$
\begin{aligned}
& \sum_{j=2}^{s+1} \int_{\Sigma_{+}}\left|\partial_{t}^{j} \partial_{x}^{s-j+1} w\right|^{2}+\sum_{j=2}^{s+1} \int_{\Omega}\left|\partial_{t}^{j} \partial_{x}^{s-j+1} w\right|^{2} \\
\leq & C\left\{\int_{\Sigma_{+}}|f|_{s-1}^{2}+\int_{\Omega}|f|_{s-1}^{2}\right\}+C\left\{\int_{\Sigma_{+}}|w|_{s}^{2}+\int_{\Omega}|w|_{s}^{2}\right\} \\
& +C\left\{\int_{\Sigma_{+}}\left|\partial_{x}^{s} w\right|_{1}^{2}+\int_{\Omega}\left|\partial_{x}^{s} w\right|_{1}^{2}\right\} .
\end{aligned}
$$

Combining (4.15) and (4.17), we get

$$
\begin{aligned}
& \sum_{j=0}^{s+1} \int_{\Sigma_{+}}\left|\partial_{t}^{j} \partial_{x}^{s-j+1} w\right|^{2}+\sum_{j=0}^{s+1} \int_{\Omega}\left|\partial_{t}^{j} \partial_{x}^{s-j+1} w\right|^{2} \\
\leq & C\left\{\int_{\Sigma_{-}}|w|_{s+2}^{2}+\int_{\Sigma_{+}}|f|_{s-1}^{2}+\int_{\Omega}\left(|f|_{(0, s+1)}^{2}+|f|_{s-1}^{2}\right)\right\} \\
& +C\left\{\int_{\Sigma_{+}}|w|_{s}^{2}+\int_{\Omega}|w|_{s}^{2}\right\} .
\end{aligned}
$$

Note that

$$
|w|_{s+1}^{2}=\sum_{j=0}^{s+1}\left|\partial_{t}^{j} \partial_{x}^{s+1-j} w\right|^{2}+|w|_{s}^{2} .
$$


It follows that

$$
\begin{aligned}
\int_{\Sigma_{+}}|w|_{s+1}^{2}+\int_{\Omega}|w|_{s+1}^{2} \leq & C\left\{\int_{\Sigma_{-}}|w|_{s+2}^{2}+\int_{\Sigma_{+}}|f|_{s-1}^{2}+\int_{\Omega}|f|_{s+1}^{2}\right\} \\
& +C\left\{\int_{\Sigma_{+}}|w|_{s}^{2}+\int_{\Omega}|w|_{s}^{2}\right\} .
\end{aligned}
$$

We finish the proof of 4.12$)(s+1)$ by the hypothesis on induction.

Remark. If $\Sigma_{-} \subset\{t=0\}$, then in (4.12) the term

$$
\int_{\Sigma_{-}}|w|_{s+1}^{2}
$$

can be replaced by

$$
\int_{\Sigma_{-}}\left(|w|_{(0, s+1)}^{2}+\left|\partial_{t} w\right|_{(0, s)}^{2}\right)
$$

It follows by checking the proof of Lemmas 4.1, 4.2 and 4.3 This fact will be used in the next section.

\section{A PRiori estimates For the CAUChy PROBlem}

In this section, we proceed to study a Cauchy problem, defined in $(x, t) \in \mathbb{R}^{n} \times$ $(0, T)$,

$$
\partial_{t t} w-k^{2}(x, t) \sum_{i, j=1}^{n} a_{i j}(x, t) \partial_{i j} w-\sum_{i=1}^{n} b_{i}(x, t) \partial_{i} w-c(x, t) w=f
$$

with

$$
w(x, 0)=w_{0}(x), \partial_{t} w(x, 0)=\tilde{w}_{0}(x) .
$$

We shall derive estimates of solutions independent of inf $k$. We assume all solutions have compact support in $x$.

We first assume that $\mathbb{R}^{n} \times(0, T)$ has the following finite decomposition:

$$
\mathbb{R}^{n} \times(0, T)=\bigcup_{l} \Omega_{l},
$$

where $\Omega_{l}$ is defined as

$$
\Omega_{l}=\left\{(x, t) ; x \in D_{l}, t_{l}^{-}(x)<t<t_{l}^{+}(x)\right\},
$$

for some domain $D_{l} \subset \mathbb{R}^{n}$ with a piecewise smooth boundary and for some piecewise smooth functions $t=t_{l}^{ \pm}(x)$ with

$$
\begin{aligned}
& 0 \leq t_{l}^{-}(x)<t_{l}^{+}(x) \leq T \text { for } x \in D_{l}, \\
& t_{l}^{-}(x)=t_{l}^{+}(x) \text { for } x \in \partial D_{l},
\end{aligned}
$$

and

$$
\left|\partial t_{l}^{ \pm}(x)\right| \leq \eta_{0} \text { for } x \in D_{l}
$$


for some positive constant $\eta_{0}$. We let $\Sigma_{l}^{ \pm}$be two surfaces over $D_{l}$ defined by $t=t_{l}^{ \pm}(x)$. We also assume for any $l$

$$
\begin{aligned}
\eta_{1} & \leq \frac{1}{\sqrt{1+\left|\partial_{x} t_{l}^{ \pm}\right|^{2}}}\left(1-k\left(\sum_{i, j=1}^{n} a_{i j} \partial_{i} t_{l}^{ \pm} \partial_{j} t_{l}^{ \pm}\right)^{\frac{1}{2}}\right) \\
& \leq \frac{1}{\sqrt{1+\left|\partial_{x} t_{l}^{ \pm}\right|^{2}}}\left(1+k\left(\sum_{i, j=1}^{n} a_{i j} \partial_{i} t_{l}^{ \pm} \partial_{j} t_{l}^{ \pm}\right)^{\frac{1}{2}}\right) \leq \eta_{2} \quad \text { on } \Sigma_{l}^{ \pm}
\end{aligned}
$$

for some positive constants $\eta_{1}$ and $\eta_{2}$. Last, we assume $\partial_{t} k^{2}$ has a fixed sign in $\Omega_{l}$ for any $l$, i.e.,

$$
\partial_{t} k^{2} \geq \text { or } \leq 0 \text { in } \Omega_{l} .
$$

Now, we rearrange $\Omega_{l}$ in the following way. Note by (5.4) that the boundary of each $\Omega_{l}$ consists of two parts $\Sigma_{l}^{+}$and $\Sigma_{l}^{-}$, which we call the upper boundary and the lower boundary of $\Omega_{l}$. First, consider those $\Omega_{l}$ whose upper boundary $\Sigma_{l}^{+}$is contained in $\{t=T\}$, i.e.,

$$
\Sigma_{l}^{+} \subset\{t=T\}
$$

and label those as $\Omega_{0,1}, \Omega_{0,2}, \cdots$. Set

$$
\omega_{0}=\bigcup_{l} \Omega_{0, l}
$$

and

$$
\sigma_{0}^{ \pm}=\bigcup_{l} \Sigma_{0, l}^{ \pm}
$$

Next, for the remaining $\Omega_{l}$, consider those $\Omega_{l}$ whose upper boundary $\Sigma_{l}^{+}$is contained in the union of $\{t=T\}$ and the lower boundaries of $\Omega_{0,1}, \Omega_{0,2}, \cdots$, i.e.,

$$
\Sigma_{l}^{+} \subset\{t=T\} \cup \sigma_{0}^{-},
$$

and label those as $\Omega_{1,1}, \Omega_{1,2}, \cdots$. Set

$$
\omega_{1}=\bigcup_{l} \Omega_{1, l}
$$

and

$$
\sigma_{1}^{ \pm}=\bigcup_{l} \Sigma_{1, l}^{ \pm}
$$

Then, consider the remaining $\Omega_{l}$ whose upper boundary $\Sigma_{l}^{+}$is contained in the union of $\{t=T\}$ and the lower boundaries of $\Omega_{0,1}, \Omega_{0,2}, \cdots$ and $\Omega_{1,1}, \Omega_{1,2}, \cdots$, i.e.,

$$
\Sigma_{l}^{+} \subset\{t=T\} \cup \sigma_{0}^{-} \cup \sigma_{1}^{-},
$$

and continue this process. The finiteness assumption implies that after finitely many steps, say at the $N$-th step, the whole collection of $\left\{\Omega_{l}\right\}$ will be exhausted. In other words, we have

$$
\mathbb{R}^{n} \times(0, T)=\bigcup_{l=0}^{N} \omega_{l}
$$


It is easy to see that

$$
\begin{aligned}
& \sigma_{l}^{-} \subset \bigcup_{j=l+1}^{N} \sigma_{j}^{+} \bigcup\{t=0\}, \quad l=0,1, \cdots, N-1, \\
& \sigma_{N}^{-} \subset\{t=0\} .
\end{aligned}
$$

Note that the finiteness assumption in the decomposition (5.3) can be relaxed. However, the finiteness in the decomposition (5.9) is essential.

In Figure 1, we have

$$
\begin{aligned}
& \omega_{0}=\Omega_{1}, \\
& \omega_{1}=\Omega_{2} \cup \Omega_{3} \cup \Omega_{4}, \\
& \omega_{2}=\Omega_{5} \cup \Omega_{6} .
\end{aligned}
$$

This implies $N=2$. In Figure 3 , we have

$$
\omega_{i}=\Omega_{i+1}, \quad i=0,1,2 .
$$

We also have $N=2$.

In the special case when $K=K(t)$, independent of $x$, and $\partial_{t} K$ changes its sign $N$ times in $(0, T)$, we then have

$$
\mathbb{R}^{n} \times(0, T)=\bigcup_{l=0}^{N} \Omega_{l}
$$

where $\Omega_{l}$ is defined as

$$
\Omega_{l}=\left\{(x, t) ; x \in \mathbb{R}^{n}, t_{l}^{-}<t<t_{l}^{+}\right\},
$$

for some constants $0=t_{N}^{-}<t_{N}^{+}=t_{N-1}^{-}<\cdots<t_{0}^{-}<t_{0}^{+}=T$. Obviously we take $\omega_{l}=\Omega_{l}$.

Now we go back to the general case. For any integer $s \geq 0$, define

$$
\left\||w \||_{s}^{2}=\sum_{j=0}^{N} \int_{\omega_{j}}|w|_{s+j}^{2} .\right.
$$

We use $\|\cdot\|_{s}$ to denote the usual Sobolev $H^{s}$-norm in $\mathbb{R}^{n} \times(0, T)$. There holds for any $s \geq 0$

$$
\|w\|_{s} \leq\||| w \mid\|_{s} \leq\|w\|_{s+N} .
$$

Lemma 5.1. For some integer $s \geq 1$, let $w$ be a $C^{N+s+2}$ solution to (5.1) in $\mathbb{R}^{n} \times(0, T)$. Suppose (5.3)-(5.8) are satisfied. Then there holds

$$
\|\left.|w|\right|_{s} ^{2} \leq C_{s}\left(\int_{t=0}\left(|w|_{(0, s+N+1)}^{2}+\left|\partial_{t} w\right|_{(0, s+N)}^{2}+|f|_{s+N-1}^{2}\right) d x+\left.|\| f|\right|_{s} ^{2}\right),
$$

where $C_{s}$ is a positive constant independent of $w$ and $f$ and depending only on $s$, $\lambda, \Lambda, T$, the decomposition (5.3), the Lipschitz norm of $k$ and $C^{s+N}$-norms of $a_{i j}$, $b_{i}, c$ and $k^{2}$ in $\mathbb{R}^{n} \times(0, T)$.

Proof. We fix the integer $s \geq 1$. We first claim that for $j=0,1, \cdots, N$ there holds

$$
\int_{\sigma_{j}^{+}}|w|_{s+j}^{2}+\int_{\omega_{j}}|w|_{s+j}^{2} \leq c_{s}\left\{\int_{\sigma_{j}^{-}}|w|_{s+j+1}^{2}+\int_{\sigma_{j}^{+}}|f|_{s+j-2}^{2}+\int_{\omega_{j}}|f|_{s+j}^{2}\right\}
$$


where $c_{s} \geq 1$ is a positive constant independent of $w$ and $f$ and depending only on $s, \lambda, \Lambda, T$, the decomposition (5.3), the Lipschitz norm of $k$ and $C^{s+N}$-norms of $a_{i j}, b_{i}, c$ and $k^{2}$ in $\mathbb{R}^{n} \times(0, T)$. Here we denote $|f|_{-1}=0$. In fact, for each $j=0,1, \cdots, N$, we have

$$
\omega_{j}=\bigcup_{l} \Omega_{j, l}
$$

and $\partial_{t} k^{2}$ has a fixed sign in each $\Omega_{j, l}$. We simply apply Lemma 4.3 with $s$ replaced by $s+j$ in $\Omega_{j, l}$ and then sum over $l$ to get (5.12).

Our goal is to take an appropriate linear combination of $(5.12) j, j=0,1, \cdots, N$, to get rid of

$$
\int_{\sigma_{j}^{-} \backslash\{t=0\}}|w|_{s+j+1}^{2}
$$

in the right side of $(5.12) j$. Note that (5.10) implies for each $j=0,1, \cdots, N-1$

$$
\begin{aligned}
\int_{\sigma_{j}^{-}}|w|_{s+j+1}^{2} & \leq \sum_{i=j+1}^{N} \int_{\sigma_{j}^{-} \cap \sigma_{i}^{+}}|w|_{s+j+1}^{2}+\int_{\sigma_{j}^{-} \cap\{t=0\}}|w|_{s+j+1}^{2} \\
& \leq \sum_{i=j+1}^{N} \int_{\sigma_{j}^{-} \cap \sigma_{i}^{+}}|w|_{s+i}^{2}+\int_{\sigma_{j}^{-} \cap\{t=0\}}|w|_{s+N}^{2} .
\end{aligned}
$$

Multiplying $(5.12)_{0},(5.12)_{1}, \cdots,(5.12)_{N}$ respectively by $1, c_{s}, c_{s}^{2}, \cdots, c_{s}^{N}$ and summing them together, we obtain with (5.13)

$$
\sum_{j=0}^{N} \int_{\omega_{j}}|w|_{s+j}^{2} \leq C\left\{\int_{\{t=0\}}|w|_{s+N+1}^{2}+\sum_{j=0}^{N} \int_{\sigma_{j}^{+}}|f|_{s+j-2}^{2}+\sum_{j=0}^{N} \int_{\omega_{j}}|f|_{s+j}^{2}\right\}
$$

for some constant $C$. By the remark at the end of the previous section, we may replace the integrand $|w|_{s+N+1}^{2}$ in the integral over $\{t=0\}$ by

$$
|w|_{(0, s+N+1)}^{2}+\left|\partial_{t} w\right|_{(0, s+N)}^{2} .
$$

Hence, we obtain

$$
\begin{gathered}
\sum_{j=0}^{N} \int_{\omega_{j}}|w|_{s+j}^{2} \leq C\left\{\int_{t=0}\left(|w|_{(0, s+N+1)}^{2}+\left|\partial_{t} w\right|_{(0, s+N)}^{2}\right)\right. \\
\left.\quad+\sum_{j=0}^{N} \int_{\sigma_{j}^{+}}|f|_{s+j-2}^{2}+\sum_{j=0}^{N} \int_{\omega_{j}}|f|_{s+j}^{2}\right\} .
\end{gathered}
$$

For integrals of $f$ over $\sigma_{j}^{+}$, we consider

$$
|f|_{s+j-2}^{2}(x, t) \leq|f|_{s+j-2}^{2}(x, 0)+2 \int_{0}^{t}|f|_{s+j-1}(x, \tau)|f|_{s+j-2}(x, \tau) d \tau
$$


which implies

$$
\begin{aligned}
\int_{\sigma_{j}^{+}}|f|_{s+j-2}^{2} & \leq \int_{t=0}|f|_{s+j-2}^{2} d x+2 \sum_{i=j}^{N} \int_{\omega_{i}}|f|_{s+j-1}|f|_{s+j-2} \\
& \leq C\left(\int_{t=0}|f|_{s+j-2}^{2} d x+\sum_{i=j}^{N} \int_{\omega_{i}}|f|_{s+i-1}^{2}\right)
\end{aligned}
$$

for some constant $C>2$. By substituting (5.16) in (5.15), we finish the proof.

Next we state Lemma 5.1 in terms of the usual Sobolev norms. In the following result, we use $\|w\|_{s}$ to denote the $H^{s}$ norm of $w$ in $\mathbb{R}^{n} \times(0, T)$ and $\|w(0)\|_{s}$ the $H^{s}$ norm of $w(\cdot, 0)$ in $t=0$.

Theorem 5.2. Suppose (5.3)-(5.8) are satisfied. Let $s \geq 1$ be an integer and let $w$ be a $C^{N+s+2}$ solution to (5.1) (5.2) in $\Omega$, with $w_{0} \in H^{s+N+1}$ and $\tilde{w} \in H^{s+N}$. Then, there holds

$$
\|w\|_{s} \leq C_{s}\left(\|w(0)\|_{s+N+1}+\left\|\partial_{t} w(0)\right\|_{s+N}+\|f\|_{s+N}\right),
$$

where $C_{s}$ is a positive constant independent of $f$ and $w$ and depending only on $s$, $\lambda, \Lambda, T$, the decomposition (5.3), the Lipschitz norm of $k$ and $C^{s+N}$-norms of $a_{i j}$, $b_{i}, c$ and $k^{2}$ in $\mathbb{R}^{n} \times(0, T)$.

Proof. For $s \geq 1$, we obtain by using the usual Sobolev norm in (5.11)

$$
\|w\|_{s}^{2} \leq c_{s}\left(\int_{t=0}\left(|w|_{(0, s+N+1)}^{2}+\left|\partial_{t} w\right|_{(0, s+N)}^{2}+|f|_{s+N-2}^{2}\right) d x+\|f\|_{s+N}^{2}\right) .
$$

By applying the trace theorem to $f$, we obtain (5.17).

As (5.17) shows, there is a loss of derivatives in the solution. This is due to the degeneracy of the hyperbolic equation. The loss is related to how many times $\partial_{t} k$ changes its sign. The importance of Theorem 5.2 is that $C_{s}$ in (5.17) does not depend on the lower bound of $k$.

Remark. In Lemma 5.1 and Theorem 5.2, the constant $C_{s}$ depends on, among others, $C^{s+N}$-norms of $a_{i j}, b_{i}, c$ and $k^{2}$ in $\mathbb{R}^{n} \times(0, T)$. In fact, we can prove that it only depends on $H^{s+N}$-norms of $a_{i j}, b_{i}, c$ and $k^{2}$ in $\mathbb{R}^{n} \times(0, T)$ if $s$ is sufficiently large, say $s \geq n+4$. To do this, we need to employ interpolation inequalities in the course of the proof. The improved dependence of $C_{s}$ on the $H^{s+N}$-norms of coefficients is important in the discussion of nonlinear hyperbolic equations (1.11). This is because we need uniform estimates in the Sobolev norms in the iteration process.

Now we can finish the proof of Theorem 1.1 .

Proof of Theorem 1.1. We assume $f$ has a compact support in $x$. The general case follows easily by an approximation. We have assumed that $a_{i j}, b_{i}, c$ and $K$ have bounded $H^{s}$-norms in $\mathbb{R}^{n} \times(0, T)$ for any positive integer $s$.

For any small $\varepsilon>0$, we consider the regularized equation in $\mathbb{R}^{n} \times(0, T)$

$$
\partial_{t t} w-(K(x, t)+\varepsilon) \sum_{i, j=1}^{n} a_{i j}(x, t) \partial_{i j} w-\sum_{i=1}^{n} b_{i}(x, t) \partial_{i} w-c(x, t) w=f,
$$


with the condition

$$
w(x, 0)=w_{0}(x), \quad \partial_{t} w(x, 0)=\tilde{w}_{0}(x) .
$$

The equation (5.18) is a strictly hyperbolic equation with smooth coefficients, and hence always admits a smooth solution $w_{\varepsilon}$ satisfying (5.19). Note that $f$ is of compact support in $x$. Hence, by the property of the propagation at finite speed, the solution $w_{\varepsilon}$ is also of compact support in $x$. Moreover, by Theorem [5.2, there holds for any integer $s \geq 1$

$$
\left\|w_{\varepsilon}\right\|_{s} \leq C_{s}\left(\left\|w_{0}\right\|_{s+N+1}+\left\|\tilde{w}_{0}\right\|_{s+N}+\|f\|_{s+N}\right),
$$

where $C_{s}$ is independent of $\varepsilon$. By a standard argument, there exists a sequence $\varepsilon_{i} \rightarrow 0$ and a function $w$ such that for any integer $s \geq 1$

$$
w_{\varepsilon_{i}} \rightarrow w \quad \text { in } H^{s}\left(\mathbb{R}^{n} \times(0, T)\right) .
$$

Then $w$ is the desired solution.

\section{REFERENCES}

[1] Colombini, F., Ishida, H., Orrú, N., On the Cauchy problem for finitely degenerate hyperbolic equations of second order, Ark. Mat., 38(2000), 223-230. MR1785400 (2001g:35183)

[2] Colombini, F., Spagnolo, S., An example of a weakly hyperbolic Cauchy problem not well posed in $C^{\infty}$, Acta Math., 148(1982), 243-253. MR0666112 (83m:35085)

[3] D'Ancona, P., Trebeschi, P., On the local solvability for a nonlinear weakly hyperbolic equation with analytic coefficients, Comm. P.D.E., 26(2001), 779-811. MR.1843284 (2002d:35132)

[4] Friedrichs, K.O., Symmetric hyperbolic linear differential equations, Comm. Pure Appl. Math., 7(1954), 345-392. MR0062932 (16:44c)

[5] Friedrichs, K.O., Symmetric positive linear differential equations, Comm. Pure Appl. Math., 11(1958), 333-418. MR0100718 (20:7147)

[6] Han, Q., Hong, J.-X., Lin, C.-S., Local isometric embedding of surfaces with nonpositive Gaussian curvature, J. Diff. Geometry, 63(2003), 475-520. MR2015470 (2004i:53081)

[7] Hörmander, L., Lectures on Nonlinear Hyperbolic Differential Equation, Springer, 1996. MR:1466700 (98e:35103)

[8] Ishida, H., Odai, H., The initial value problem for some degenerate hyperbolic equations of second order in Gevrey classes, Funkcial. Ekvac., 43(2000), 71-85. MR1774369|(2001f:35281)

[9] Ishida, H., Yagdjian, K., On a sharp Levi condition in Gevrey classes for some infinitely degenerate hyperbolic equations and its necessity, Publ. Res. Inst. Math. Sci. 38(2002), 265287. MR1903740(2003e:35174)

[10] Kajitani, K., Nishitani, T., The Hyperbolic Cauchy Problem, Lecture Notes in Math., 1505, Springer-Verlag, Berlin-Heidelberg-New York, 1991. MR.1166190 (94f:35074)

[11] Yagdjian, K., The Cauchy Problem for Hyperbolic Operators: Multiple Characteristics. Micro-Local Approach, Math. Top., 12, Akademie Verlag, Berlin, 1997. MR:1469977 (98h:35145)

Department of Mathematics, University of Notre Dame, Notre Dame, Indiana 46556 And - Max-Planck Institute for Mathematics, Inselstr. 22 - 26, 04103 Leipzig, Germany

E-mail address: qhan@nd.edu, qinghan@mis.mpg.de

Institute of Mathematics, Fudan University, Shanghai, People's Republic of China

E-mail address: jxhong@fudan.ac.cn

Department of Mathematics, National Chung-Cheng University, Ming-Hsiung, Chiayi, TAIWAN

E-mail address: cslin@math.ccu.edu.tw 